\title{
INFRAESTRUCTURA E INFOCULTURA DE LAS TIL EN LA GESTIÓN UNIVERSITARIA: UPEL-IMPM Extensión Académica Paraguaná
}

Uriel José Castellanos Aguirre

\section{Resumen}

El objetivo fue interpretar los desafíos de la UPEL-IMPM Extensión Académica Paraguaná en la incorporación de las Tecnologías de Información Libre (TIL). El diseño de la investigación se fundamentó en el paradigma mixto. Fue cuantitativo al determinar las percepciones de los discentes respecto a la infoestructura tecnológica y cualitativo al indagar la Infocultura de los gestores sobre la incorporación de las TIL. Los hallazgos nos permitieron evidenciar el uso de las TIC en las actividades cotidianas, pero aún es incipiente. Los desafíos en la Infoestructura e Infocultura obligan a repensar la gestión académica para atender las necesidades de todos los actores involucrados, ya que el uso de las TIL en el cotidiano, desde la creación de información digital hasta su comunicación, nos permitirá ampliar y (re)configurar nuestras prácticas sociales y culturales, a pesar de los problemas políticos, económicos y sociales en los servicios públicos y de acceso a internet.

Palabras clave: Educación; Software Libre; Gestión Académica; TIC; Políticas Educativas.

\section{INFRAESTRUCTURA E INFOCULTURA DAS TIL NA GESTÃO DA UNIVERSIDADE: UPEL-IMPM Extensión Académica Paraguaná}

Resumo

O objetivo foi interpretar os desafios da Extensão Acadêmica UPEL-IMPM Paraguaná na incorporação das Tecnologias de Informação Livres (TIL). O projeto de pesquisa foi baseado no paradigma misto. Foi quantitativo ao determinar as percepções dos discentes quanto à infoestrutura tecnológica e qualitativo ao investigar a Infocultura dos gestores sobre a incorporação do TIL. Os achados permitiram mostrar que as TIC são utilizadas nas atividades universitárias, mas ainda são incipientes. Os desafios da Infraestrutura e da Infocultura nos obrigam a repensar a gestão acadêmica para atender às necessidades de todos os atores envolvidos, pois o uso do TIL no cotidiano, desde a criação da informação digital até a sua comunicação, nos permitirá expandir e (re)configurar nossas práticas sociais e culturais. No entanto, devido aos diversos problemas políticos, econômicos e sociais do país, expostos pela insatisfação com os serviços públicos e com o acesso à internet, seus benefícios não são explorados.

Palavras-chave: Educação; Software Livre; Gestão acadêmica; TIC; Políticas educacionais.

\section{INFRASTRUCTURE AND INFOCULTURE OF TIL IN UNIVERSITY MANAGEMENT: \\ UPEL-IMPM Extensión Académica Paraguaná}

\section{Abstract}

The objective was to interpret the challenges of the UPEL-IMPM Academic Extension Paraguaná in the incorporation of Free Information Technologies (Tecnologías de Información Libre, TIL). The research design was based on the mixed paradigm. It was quantitative when determining the perceptions of the students regarding the technological infostructure and qualitative when investigating the Infoculture of the managers 
DOI: $10.12957 /$ teias. $\%$ Y.55438

about the incorporation of the TIL. The findings allowed us to demonstrate the use of ICT in daily activities, but it is still incipient. The challenges in the Infrastructure and Infoculture force us to rethink academic management to meet the needs of all the actors involved, since the use of TIL in daily life, from the creation of digital information to its communication, will allow us to expand and (re)configure our social and cultural practices, despite political, economic and social problems in public services and internet access.

Keywords: Education; Free Software; Academic management; ICT; Educational policies.

\section{INTRODUCCIÓN}

La incorporación de las Tecnologías de la Información y Comunicación (TIC), hoy tecnologías digitales (BONILLA, 2012), en las Instituciones de Educación Superior (IES) de Venezuela son requeridas cada día más en las actividades académicas, administrativas y de extensión por lo que debemos cuestionar estos escenarios para mantener un equilibrio en la relaciones simbiótica que repercuten en aspectos sociales, políticos, económicos y culturales. Requerimos conocimientos y habilidades que promuevan el acceso, creación, uso, modificación, publicación y distribución de las informaciones con el uso de las tecnologías digitales a través de la motivación y la creatividad. Para lo cual debemos garantizar actividades cotidianas en las que se envuelvan reflexiones teóricas, pedagógicas y tecnológicas con nuestras particularidades culturales y sociales (PRETTO, 2013, 2017).

La Universidad Pedagógica Experimental Libertador - Instituto de Mejoramiento Profesional del Magisterio, en su Extensión Académica Paraguaná (UPEL-IMPM Extensión Académica Paraguaná), ubicada en Los Taques, Punto Fijo Estado Falcón, Venezuela, continua en la búsqueda de garantizar la innovación mediante el uso de las tecnologías digitales junto a la apropiación de la Ley de Infogobierno (VENEZUELA, 2013), por lo que promueve estudios sobre las Tecnologías de la Información Libres (TIL). Este acrónimo "TIL”, fue desarrollado por estado venezolano para identificar:

[...] aquellas tecnologías con estándares abiertos que garantizan el acceso a todo el código fuente y la transferencia del conocimiento asociado para su comprensión; libertad de modificación; libertad de uso en cualquier área, aplicación o propósito y libertad de publicación del código fuente y sus modificaciones. (VENEZUELA, 2013, p. 4)

Pero implementar el uso de Software Libre en Venezuela representa un desafío, ya que no es cuestión del uso técnico de aplicativos, sino que también se debe adoptar una posición respecto a la filosofía del Software Libre, proceso en el que la Extensión Académica, para el 2020, aun en época de pandemia, no había iniciado tanto en su capacitación como su migración, además de requerir receptividad en los ambientes educativos.

La obligatoriedad a las universidades en asumir el proceso de migración al Software Libre y datos abiertos (VENEZUELA, 2013, p. 4), representa diferentes desafíos debido a: los retrasos en los procedimientos y ejecución a partir de notificaciones emanadas por el Ministerio del Poder Popular para la Educación Universitaria Ciencia, Tecnología (MPPEUCT); y, que el Estado no expone datos abiertos sobre la situación actual del proceso de migración en las IEU, las últimas expuestas por el Centro Nacional de Tecnología de la Información (CNTI), datan del 2012, documento que expone estadísticas de Migración en la Administración Pública Nacional (2012), en estas se consolidan los datos de 203 instituciones públicas, pero no discrimina la situación en las universidades. En este documento se expone que, para la época, solo el 51,14\% usaba el Sistema 
Operativo (SO) Canaima, distribución Linux/GNU desarrollada por el Estado (CASTELLANOSAGUIRRE; PRETTOO, 2019).

Bonilla y Pretto (2015), expresan que los desafíos que debemos asumir al implementar el Software Libre y los datos abiertos parten "[...] de la articulación colectiva, colaborativa y abierta, en un momento que aún estamos presos a modelos cerrados e individualistas, lamentablemente tenemos como referencia para la educación la llamada lógica del mercado.” (p. 16, traducción nuestra $)^{1}$. Es por este motivo que al abordar la filosofía del Software Libre y los datos abiertos buscamos reducir las brechas sociales, al mismo tiempo en que colaboramos a enfrentar las dificultades en los procesos llevados a cabo por algunas instituciones, inicialmente sobre el nivel técnico. Esto es debido a que se demanda por parte de todos los involucrados el dominio de competencias específicas en los escenarios educativos relacionadas con el uso de las tecnologías. Sobre estas consideraciones, pretendemos interpretar los desafios de la UPEL-IMPM Extensión Académica Paraguaná en la incorporación de las TIL.

\section{METODOLOGÍA}

Para el siguiente estudio, adoptamos un abordaje mixto. Partimos del enfoque cuantitativo para determinar las percepciones de los discentes respecto a la infoestructura tecnológica de la Extensión Académica; la unidad de análisis estuvo conformada a partir del muestreo no probabilístico de casos-tipo (HERNÁNDEZ, FERNÁNDEZ Y BAPTISTA, 2010), donde se tomó como criterios que fuesen: a. Estudiante regular de la Extensión Académica, y b. Activo en el Período Académico. Se obtuvo una muestra intencional de sesenta y cinco (65) discentes los cuales se consideraron apropiados para el estudio del caso, debido a que la población total de la extensión universitaria, para el momento del estudio, era de 200 discentes. Lo que nos permite obtener una dimensión considerable de opiniones sobre las percepciones para establecer un dialogo sobre el comportamiento dentro de la Extensión Académica, lo cual ofrece riqueza, profundidad y calidad a nuestro enfoque de estudio. La técnica para colectar las informaciones fue la encuesta. Se utilizó un cuestionario tipo escala de Likert formado por veinticuatro (24) ítems con las siguientes opciones de respuesta: (TA=Totalmente de acuerdo, 5; DA=De acuerdo, 4; I=Indeciso, 3; $\mathrm{ED}=$ En desacuerdo, 2; TD=Total desacuerdo, 1). La validez de la información fue generada por tres (3) expertos en el área. El Coeficiente de Confiabilidad se calculó mediante una prueba piloto a quince (15) estudiantes en donde el alfa de Cronbach arrojo 0,956 puntos, al igual que la consistencia interna de las variables, calificándose como "muy alto" (RUIZ, 2002, p. 39). Finalmente, se procedió al análisis de la información obtenida, al organizar las informaciones en un software de datos (Libre Office Calc) y luego procesarlos (mediante el Software SPSS v. 18.0) que permitieron obtener las tablas y figuras a ser analizados.

Para el enfoque cualitativo, indagamos la Infocultura de los gestores de la Extensión Académica sobre la incorporación de las TIL, nuestro diseño de campo tuvo la influencia del método Hermenéutico-interpretativo (SANDOVAL, 2002), en donde se interpretaron las informaciones respecto a las variables conocimiento, uso, importancia e incorporación a las actividades laborales (RAMÍREZ, 2009). La técnica para la recolección de las informaciones que utilizamos, fue la entrevista a profundidad, a partir de un guion de entrevista constituido por quince (15) preguntas abiertas, diseñado a partir de los aspectos que permitieron profundizar la

$1[\ldots]$ da articulação coletiva, colaborativa, aberta, num momento que ainda estamos presos a modelos fechados e individualistas, lamentavelmente tendo como referência para a educação a chamada lógica do mercado. 
Infocultura. La validez y confiabilidad radicó en el esfuerzo por realizar un retrato denso de las situaciones que relatan y expresan tanto los sujetos observados como la teoría expuesta. La ética para la investigación tiene respaldo en un término de consentimiento libre, en donde los informantes claves aceptaron participar. La selección de los informantes clave estuvo bajo los criterios de ser: a. Personal docente y administrativo de la Extensión Académica; b. Desear participar; y, c. Ser personal activo; lo que resulto en siete participantes. Las informaciones se procesaron utilizando el Software ATLAS/ti, versión 7.5, que nos permitieron elaborar los análisis posteriores.

\section{TIL Y LA GESTIÓN UNIVERSITARIA}

Las Tecnologías de la Información Libres (TIL) posibilitan una nueva estructura de comunicación y organización que permiten la implementación de las tecnologías digitales con una filosofía del Software Libre y datos abiertos (VENEZUELA, 2013). Estas nuevas relaciones con el software, a través de una interfaz y una pantalla como expresión tangible nos lleva a la simulación de lo real, dinámica que esta contenida en la cultura digital (MONTOYA-GONZÁLEZ; BONILLA, 2019). Por ello, las TIL posibilitan la integración de entornos en las prácticas del cotidiano, en las que se permite el acceso, creación, uso, modificación, publicación y distribución de medios digitales (textos, imágenes, audios, videos o elementos interactivos combinados) mediante la red de internet (BONILLA; PRETTO, 2015). Ramírez (2009), nos lleva a reflexionar que "las innovaciones tecnológicas requieren ambientes receptivos." (p. 63), los cuales nos posibilitan enfrenar los desafíos mediante la alineación y expectativas de los grupos sociales involucrados.

Por esta razón, la percepción que consideramos en este estudio parte de la flexibilidad en los ámbitos de creación, transmisión y difusión del conocimiento, así como la reconfiguración en los roles de los docentes y discentes. Córdova, Muñoz-Cano y Priego (2011) asumen cinco funciones que permiten elaborar este estudio, las cuales son: a. Programa de la asignatura; b. Materiales de apoyo; c. Participación del docente; d. Participación del estudiante; y finalmente, e. Conocimiento sobre las TIC. Las funciones anteriores nos guiaron en nuestro propósito, pues se requiere de un trabajo donde no sólo se reproduzca, imite o simule, sino en el cual se innove con la presencia de las TIL aunado a la participación del docente. De modo que, formar competencias, en TIL, debe ser parte de la educación, pero resulta complejo pues aún no se fomenta adecuadamente (BONILLA; PRETTO, 2015), sin embargo, los grupos universitarios tienen que plegarse a las exigencias de la Ley de Infogobierno (VENEZUELA, 2013).

Nos es fundamental considerar que las innovaciones educativas deben ir acompañadas de planes estratégicos, en donde los diferentes grupos universitarios se apropien de estas nuevas tecnologías. Ramírez (2009) afirma que se debe incursionar por:

[...] (1) apropiación del objeto, la tecnología es considerada en forma muy superficial, sin realmente pensar acerca de sus beneficios, aunque se conoce su importancia de contar con él, (2) apropiación de la funcionalidad, este nivel de apropiación, implica familiarizarse con el funcionamiento del recurso tecnológico, y (3) apropiación de nuevas formas de aprendizaje, este nivel es el más profundo de apropiación, que involucra usar la tecnología como una herramienta de aprendizaje para desarrollar proyectos, que sean relevantes a condiciones locales, intereses y problemas y vinculados [...]. (p.63-64)

En estas tres etapas, la apropiación del objeto, de la funcionalidad y de nuevas formas de aprendizaje con él, nos guía en la adopción, adaptación y apropiación de las tecnologías como parte 
DOI: $10.12957 /$ teias. $\%$ Y.55438

integral de las actividades cotidianas, evaluando su uso con dirección a necesidades específicas. Por lo que, para ahondar en el conocimiento, proponemos dos términos de la "Técnica, Sociedad y Cultura" del Programa de las Naciones Unidas para el Desarrollo (2002) en el uso de las TIL, los cuales son: Infoestructura e Infocultura.

Según el Programa de las Naciones Unidas para el Desarrollo (2002), tenemos que: Por un lado, la Infoestructura, es toda aquella tecnología tangible, comercializada por el hombre. Es habilitante: facilita, capacita, permite, es necesaria, pero no suficiente, sin el desarrollo correspondiente de los aspectos del estrato cultural no puede garantizarse su efectividad, esto quiere decir, que representa un elemento que por sí solo no genera procesos de desarrollo. Por otro lado, la Infocultura busca garantiza una actitud favorable que toma en cuenta a los participantes para que perciban y den uso adecuado de la infoestructura, se apropien de la tecnología y articulen el uso de las plataformas tecnológicas con el ámbito social. Para el desarrollo adecuado de las TIL desde la perspectiva de la infoestructura e infocultura, es necesario incorporar los desafíos y retos tecnológicos en conjunto al tiempo y espacio adecuado para ello. En este sentido, los procesos académicos deben considerar la flexibilidad en los ámbitos de creación, transmisión y difusión del conocimiento, así como la reconfiguración en los roles del personal administrativo y docente, en los cuales es necesario una ética backer, la cual Menezes y Pretto (2019) resaltan la pasión, creatividad y valor social en la realización de la tareas o búsqueda de soluciones a los problemas.

\section{LOS HALLAZGOS}

La población discente del estudio resultó ser heterogénea, ya que: el 56\% fueron del sexo femenino y el $44 \%$ es masculino, de los cuales el $70 \%$ de ellos poseen un computador de uso personal, el $72 \%$ tienen acceso a internet para la comunicación y realización de actividades académicas, finalmente el 96\% goza de correo electrónico para comunicarse con los docentes y compañeros de clases. Estos datos permiten afirmar que el $70 \%$ puede acceder a las actividades desde la comodidad de su hogar, ya que poseen PC personal e internet. El 30\% restante se encuentra al margen en el acceso a las actividades y al observar detenidamente solo el $4 \%$ no posee un medio de comunicación en línea (e-mail), esto es preocupante ante la pérdida de contacto. Pero, cabe señalar que se suple con la modalidad que se utiliza en la Extensión Académica para impartir clases, donde cada programa contempla horas académicas presenciales y a distancia.

Los resultados obtenidos de los veinticuatro (24) ítems, fueron organizados y analizados con el Software SPSS v. 18.0 a fin de obtener los valores medios y las desviaciones estándar. Los resultados obtenidos son presentados por ítems en la dimensiones a partir de las valoraciones ofrecidas por el grupo de discentes:

Tabla 1: Resumen de las respuestas por ítems de los estudiantes con respecto a los procesos académicos de la UPEL-IMPM Extensión Académica Paraguaná.

\begin{tabular}{lc}
\hline & \multicolumn{2}{c}{ Respuestas } \\
\cline { 2 - 2 } Indicadores & $\mathrm{X}$ Ds \\
\hline
\end{tabular}

a. Programa de asignatura

1. Desde el punto de vista científico, los diferentes contenidos que se presentan son actuales.

$3,28 \quad 1,59$


DOI: $10.12957 /$ teias. $^{\%}$ Y. 55438

2. Las características de los programa ofrecen una diversidad de recursos Tecnológicos.

3. El programa se presenta en formato digital e impreso.

4. Las actividades que ofrece el programa mediante el uso de tecnología, favorecen la

$3,14 \quad 1,6$
comprensión de los contenidos por parte del usuario.

\section{b. Materiales de apoyo}

5. Los diferentes recursos tecnológicos que se ofrecen facilitan la comprensión de la $3,26 \quad 1,76$ información.

6. Los recursos tecnológicos presentados son interesantes desde un punto de vista teórico.

7. Los recursos tecnológicos presentados son relevantes desde un punto de vista práctico.

$3,2 \quad 1,58$

8. En general, los recursos tecnológicos ofrecidos son pertinentes desde el punto de vista de la calidad científica.

\section{c. Participación del docente.}

9. El uso del e-mail entre estudiantes y docentes es pertinente.

10. El docente ofrece una diversidad de opciones que facilitan la comunicación para solicitar asesorías.

11. El docente ofrece una diversidad de opciones que facilitan la comunicación para recibir asesorías.

\begin{tabular}{lcc} 
12. El docente ofrece una diversidad de opciones que facilitan la entrega de actividades & 3,18 & 1,71 \\
\hline 13. El docente ofrece una diversidad de opciones que facilitan la retroalimentación. & 3,34 & 1,55
\end{tabular}

\section{d. Participación del estudiante}

\begin{tabular}{lcc}
\hline 14. La utilización de foros me resulta comprensible & 2,92 & 1,58 \\
\hline 15. Suelo participar en foros generales & 3,06 & 1,49 \\
\hline 16. El docente me estimula a reflexionar en foros específicos & 3,04 & 1,58 \\
\hline 17. El docente utiliza Entornos Virtuales Apoyo (EVAp) & 2,8 & 1,47 \\
\hline 18. Los Entornos Virtuales Apoyo (EVAp) lo calificaría como sencillos. & 2,8 & 1,4 \\
\hline e. Conocimiento sobre las TIC (Tecnologías de la Información y Comunicación) & 3,08 & 1,83 \\
\hline 19. Considero que las TIC son herramientas educativas. & 3,22 & 1,66
\end{tabular}


DOI: $10.12957 /$ teias. $\%$ Y.55438

21. En general, las TIC son importantes para la enseñanza.

22. Uso las TIC en actividades Educativas.

23. Uso el Software Libre en actividades Educativas.

24. Uso páginas web con el fin aprender a usar las TIC o el Software Libre

$3,1 \quad 1,74$

Fuente: Datos procesados por el autor con el Software SPSS v.18.0

Luego aplicamos una la fórmula que denominamos Índice General de Percepción del Uso de las TIC (IGPUT). Esta se alcanzó al realizar la sumatoria de manera independiente de cada una de las respuestas obtenidas por indicador entre el número de indicadores, generando así, los promedios y de ello su desviación estándar, para ser procesados posteriormente con el Software SPSS v. 18.0. Esta fórmula permitió dar resultados puntuales de la situación, explicando así, el Índice General de percepción del Uso de las TIC mediante el análisis exhaustivo de los datos.

Tabla 2: Resumen de la percepción sobre las TIC que poseen los estudiantes con respecto a los procesos académicos de la UPEL-IMPM Extensión Académica Paraguaná.

\begin{tabular}{lccccc} 
Indicador & $\mathbf{X}$ & Mayor X & Menor X & Rang & Ds \\
\hline Programa de asignatura & 3,21 & 5,0 & 1,0 & 4,0 & 1,49 \\
\hline Materiales de apoyo & 3,29 & 5,0 & 1,0 & 4,0 & 1,49 \\
\hline Participación del docente & 3,14 & 5,0 & 1,0 & 4,0 & 1,5 \\
\hline Participación del estudiante & 2,92 & 5,0 & 1,0 & 4,0 & 1,36 \\
\hline Conocimiento sobre las TIC & 3,14 & 5,0 & 1,0 & 4,0 & 1,68
\end{tabular}

Fuente: Datos procesados por el autor con el Software SPSS v.18.0

Figura 1: Resumen de la percepción sobre las TIC que poseen los estudiantes con respecto a los procesos académicos de la UPEL-IMPM Extensión Académica Paraguaná. 


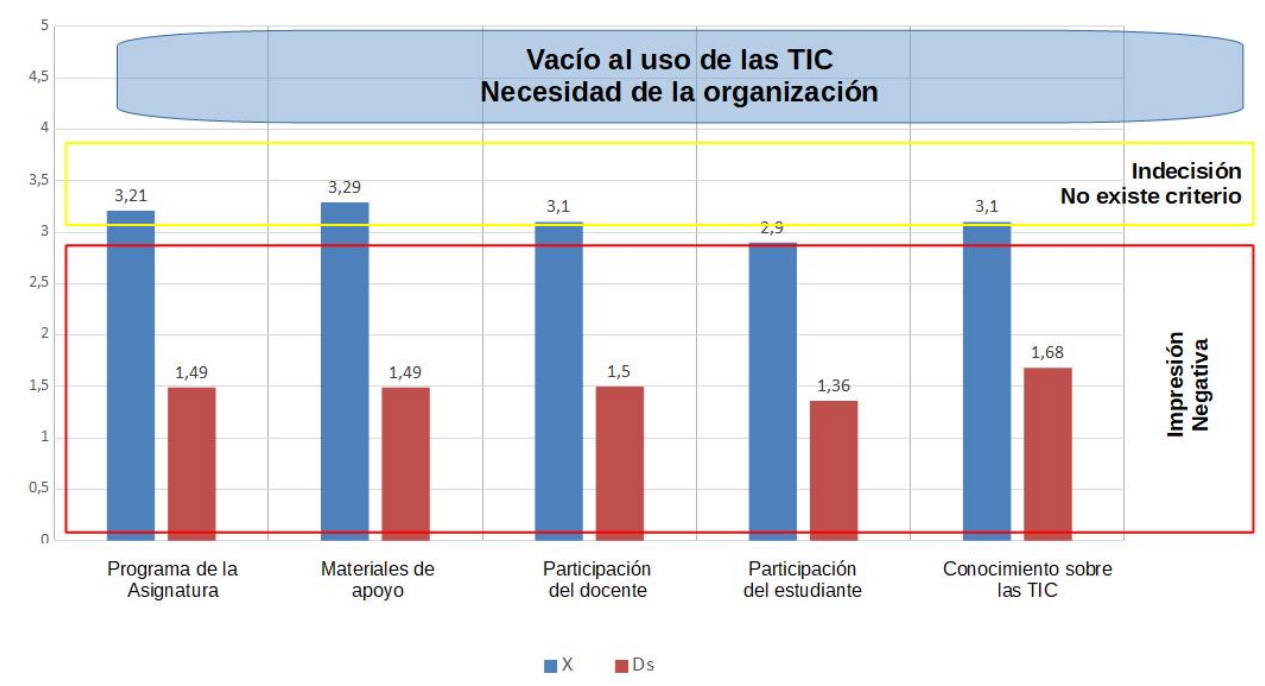

Fuente: Figura procesada por el autor.

En consecuencia, a lo observado (ver tabla 2 y figura 1), la percepción sobre las TIC que poseen los discentes con respecto a los procesos académicos en la Extensión Académica, las opiniones hacia los indicadores, fueron muy similares en términos generales y esto se concreta al observar las medias en la escala I=Indeciso (3). Señalamos que los indicadores destacados fueron el 1. Programa de la asignatura y 2. Materiales de apoyo con una media de 3,21 y 3,29 puntos cada uno, ambos con una desviación estándar de 1,49 puntos. Exteriorizando que los discentes reflexionan sobre el contexto educativo en cuanto a la forma de que se les imparte el conocimiento. Es decir, los programas y las actividades, reflejan la reproducción de una práctica educativa semejante a aquella en la cual se encuentran presentes las TIC para una institución con un sistema semi-presencial. Este ambiente fomenta que los estudiantes perciban amigables aquellas actividades que puedan colaborar económicamente en su cotidiano, además dar respuesta o soluciones con ayuda de las TIC constituye para la formación de los estudiantes autonomía que le permite ser capaz de obtener, analizar y discriminar la información que se encuentra en la red. En consecuencia se debe sustentar que en la Extensión Académica la necesidad de un proceso de alfabetización digital para el uso de la información en línea y para la construcción y aplicación de este conocimiento.

Por otra parte, los indicadores 3. Participación del docente y 5. Conocimiento sobre las TIC tienen una media igual de 3,14 puntos y una desviación estándar de 1,5 y 1,68 puntos al respecto. Valorando así los procesos de entrega de actividades y respectiva retroalimentación en el tiempo por parte del docente, aunándose que los estudiantes poseen habilidades para el uso de las tecnologías digitales en los procesos educativos (PRETTO, 2017). Sin embargo, el indicador 4. Participación del estudiante es el menos valorado, con una media de 2,92 puntos y una desviación estándar de 1,36 puntos. Acentuamos la importancia de éste, ya que los procesos de comunicación y participación de manera activa son fundamentales entre discente-docente y discente-discente, reflejando la reflexión y favoreciendo la comprensión. El estudio indica que la utilización de los medios como apoyo a los procesos pedagógicos, no obedece a una acción concebida como parte del entramado estratégico de los cursos, sino que se entiende como usos esporádicos, espontáneos o accidentales de los cuales se vale el docente para atender situaciones puntuales. De igual modo, la percepción que pueda asumirse en relación a las TIC, están vinculadas directamente con los 
DOI: $10.12957 /$ teias. $\%$ Y.55438

aprendizajes que se quieran promover y para ello la creatividad y el diseño juega un papel importante, así como las condiciones de implementación que se realizan para que sean integrados en los ambientes de aprendizaje. De la misma manera, se asume que si estas innovaciones no son acompañadas de programas educativos bien fundamentados y tratados, no va a ir más allá de brindarle a la gente nuevas aplicaciones a través de tecnologías.

Todo ello, nos lleva a observar los tres escenarios en la percepción sobre las TIC que poseen los estudiantes con respecto a los procesos académicos de la UPEL-IMPM Extensión Académica Paraguaná. Se presenta una impresión negativa considerable, con una indecisión por parte de algunos discentes, lo cual concluye con el vacío existente y acentúa claramente la necesidad de la organización hacia el uso de las tecnologías digitales.

En cuanto a las entrevistas a profundidad aplicadas a los actores universitarios, organizamos las informaciones, para examinarlas detalladamente y en profundidad, y así identificar incidentes posibles de ser codificados al punto de establecer relaciones y categorías que integraran estos elementos. De este modo generamos una figura con veintidós (22) códigos y tres (3) categorías principales, en azul, que relacionan las narrativas abordadas.

Figura 2: Códigos iniciales de análisis en la unidad académica.

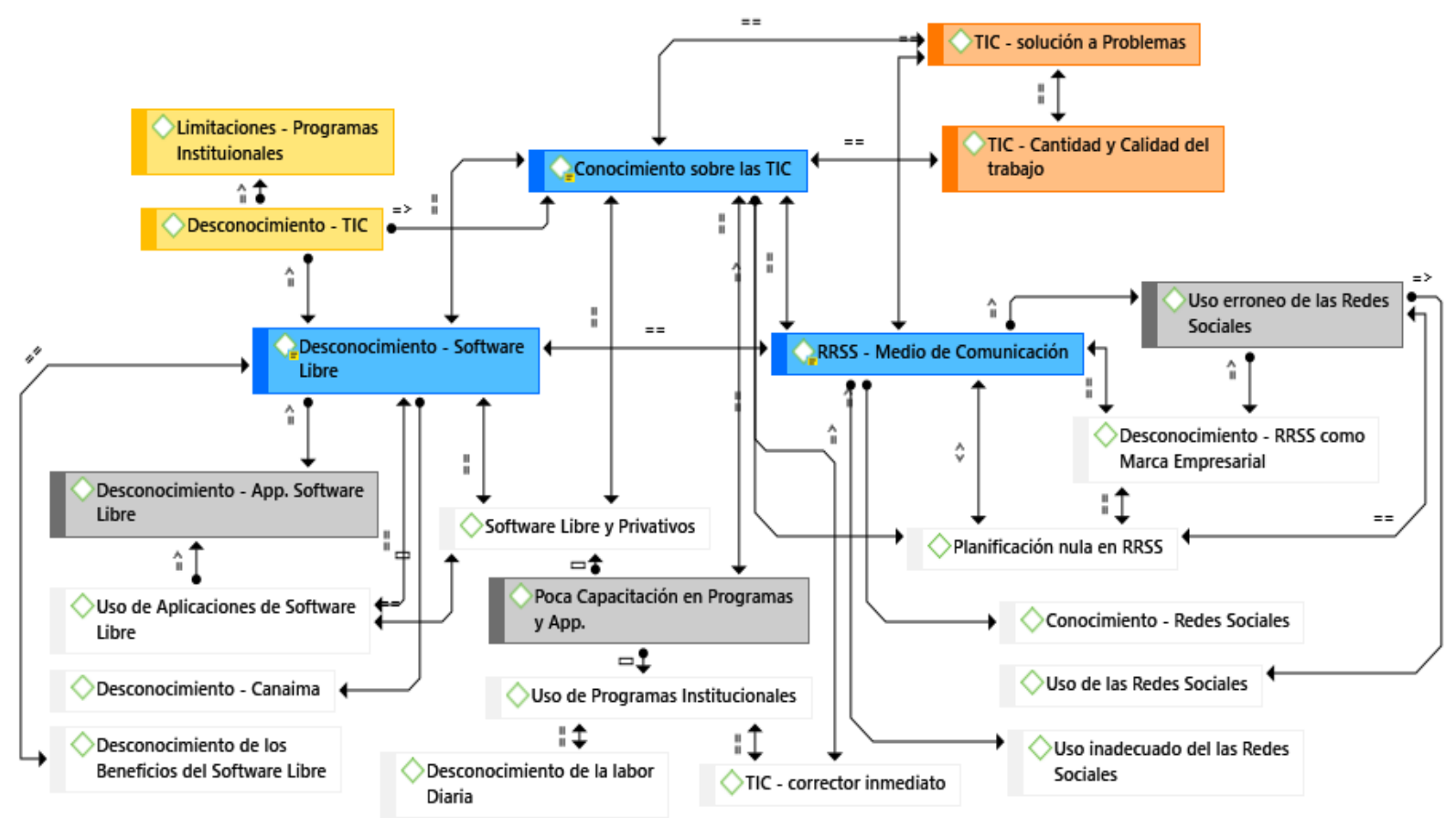

Fuente: Figura procesada por el autor en el Software ATLAS/ti, versión 7.5.

Como mencionamos anteriormente, estos datos nos permitieron generar tres categorías principales, al identificar algunos elementos estructurales que subyacen dentro de institución, los cuales son: a. Las TIC en la unidad académica; b. Las redes sociales en la unidad académica; y, c. El Software Libre en la unidad académica.

Inicialmente, al cuestionar sobre la Infoestructura Tecnológica presente en la Extensión Académica (su Entorno, Tipo de Conexión, Conectividad, Sistemas y Hardware y, Cuerpo docente y Administrativo), percibimos que esta es considerada como adecuada para el cumplimiento básico de las funciones. Asimismo, los informantes manifestaron el uso limitado y poco sistemático de las 
tecnologías por el tipo de conexión a internet desde sus hogares y/o dispositivos móviles, presentan constantes interrupciones, lo que la sitúa en un escenario poco competitivo en comparación con otras instituciones de su misma condición. Pretto (2017) manifiesta que estos escenarios de adopción de las tecnologías digitales, necesitan construirse a partir de los problemas y obstáculos que encontramos en nuestro cotidiano, donde se valorice el error, y a partir de él, construir y desafiar la creación de conocimiento no solo intelectual, sino sobre nuestras culturas. En consecuencia, es de carácter emergente que auspicie alternativas ante esta problemática detectada en la extensión y así lograr la utilización completa de las tecnologías digitales como apoyo a la ejecución de los procesos Universitarios. Además, al igual que Menezes y Pretto (2019), consideramos que abordar el escenario técnico para conocer y modificar su naturaleza, nos permite (re)significar las tecnologías digitales; contexto necesario, debido que estas tecnologías que adaptamos a nuestros espacios educativos, no siempre son diseñadas para tal fin. Lo que genera las bases para que los productos científicos y culturales creados, puedan ser apropiados por todos a partir de sus potencialidades y desafíos que estos traen a nuestro día a día.

Respecto al uso de las TIC en la Extensión Académica, evidenciamos que las actividades diarias de los trabajadores administrativos y docentes, asumen procesos repetitivos a cada cierto periodo de tiempo, común en estos escenarios. Los diferentes actores mencionaron que la realización de las actividades queda limitada al conocimiento del operador de los softwares o aplicativos, lo que condiciona el tiempo del personal en la ejecución de la actividad. De modo que, se hace necesario una mayor formación para disminuir las dificultades o retrasos en la ejecución de las actividades, escenario que Bonilla y Pretto (2015) manifiestan que pueden ser abordados a partir de la colaboración; en la cual acrecentamos que se deben dar de manera espontanea tanto dentro como con otros departamentos que realicen actividades semejantes. Asimismo, el personal administrativo manifestó que el uso de los softwares de ofimática privativo (Microsoft Officeß) pueden ser sustituidas por softwares específicos desarrollados por la Extensión Académica para elaborar sus actividades, sin embargo, no tiene la formación y o el respaldo para ello. En cuanto a los profesores, señalan la necesidad formación digital para innovar en sus aulas de clase, lo que genera oportunidades para elaborar programas de formación que aborden esta temática.

En este contexto, se hace necesario explorar y documentar experiencias locales para establecer análisis crítico a los medios de comunicación, al mismo tiempo, esta información colectada posibilitara divulgar las mejores opciones de software o aplicativos para la Extensión Académica, estudiantes y la población en general, logrando así que se comprometan con los medios digitales, utilizando las herramientas para la libertad de expresión, el pluralismo, el diálogo intercultural y la tolerancia, y como ciudadanos que aportan al debate democrático para el buen gobierno dentro del ambiente laboral.

Respecto al internet, en la Extensión Académica, el personal administrativo no tiene conexión a ninguna red (interna o externa). Evidenciamos que el uso de pendriver para el intercambio de informaciones, dificultan los procesos, puesto que los virus suelen eliminar actividades ya elaboradas y retrasan los plazos aún más. Escenario que puede ser fácilmente solucionado al utilizar sistemas operativos libres, sin embargo, esta acción depende no solo de una aprobación para la ejecución institucional, sino de un plan de migración completo que incluya formación a todos los involucrados. Todo ello nos lleva a pensar en una formación que como afirman Bonilla (2012) y Pretto (2017), debe ser dirigida cada vez mas a no solo usar los programas o softwares, sino a como (re)producirlos; contexto que no lleva a discutir colectivamente las necesidades, negociar nuestras diferencias y ejecutar planos factibles de acción. Ademas, el contexto de acceso a intenet no es una novedad, ya que las estadísticas disponibles de conexión por la 
empresa internetworldstats ${ }^{2}$, estima que de 7,87 Billones de personas de la población mundial al 2020, solo el 8,4\% están conectadas en América Latina y el Caribe, lo que nos indica que no escapamos de la realidad social en la que estamos inmersos y debemos establecer acciones para fortalecer nuestras bases locales en conexión.

Por ultimo, observamos que para fines de comunicación de las informaciones con los diferentes discentes (activos y egresados), se utilizan redes sociales, en las que solo dos personas de la coordinación poseen autorización por el coordinador general. De este hecho, al cuestionar su uso, logramos percibir que las publicaciones en las redes sociales se presentan sin una planificación previa o seguimiento posterior, lo que genera descontento por parte de los discentes que participan en la red social, al comentar la falta de respuestas oportunas por parte de la Universidad. Por lo que cuestionamos que estos espacios sociales de la Extensión Académica (principalmente en el grupo de Facebook $\left.{ }^{\circledR}\right)$, que permiten una vía de comunicación directa y colaborativa con toda la comunidad de la universidad, revela lo que Castells (2017) y, Bonilla y Pretto (2015), expresan como formas de expresión en redes, las cuales deben permitir llevar información relevante a todos los que allí se encuentran.

Percibimos entonces que las redes sociales ofrecen nuevas posibilidades en los procesos de gestión, al mismo tiempo, condicionan la forma de comunicación dentro de la Extensión Académica. Lo que nos conduce a sugerir que las comunicaciones en las redes sociales contengan un seguimiento para exponer respuestas oportunas, es decir, estudiar pedagogías y protocolos sociales para establecer procesos de discernimiento. De esta manera, la Extensión Académica podrá producir, autorizarse y constituir una comunidad social en red o "sociedades en red" (Castells, 2017), que a cada día ganará más información oportuna de los gestores, al explotan todas las posibilidades y ventajas que estos ambientes ofrecen.

En este punto, es necesario repensar que las políticas públicas se adentraron a las acciones administrativas y docentes, pero percibimos que Software Libre no es prioritario para los informantes claves debido que no es representativo en sus funciones diarias. Es, por tanto, un desafío a la formación inicial y continua de todos en la unidad académica el desarrollo de programas de formación que permitan comprender la compleja relación de la cultura y la apropiación del Software Libre. Esta relación entre la cultura y el uso de las tecnologías digitales con la filosofía del Software Libre permitirá el fortalecimiento de los involucrados como creadores y productores de conocimiento, en un contexto de diversidad digital (BONILLA; PRETTTO, 2015).

\section{REFLEXIONES FINALES}

Los planteamientos anteriores nos llevan a considerar que la Extensión Académica presenta innumerables desafíos a abordar en el futuro. La Infocultura en la extensión evidencia el uso de las TIC en las actividades diarias que a pesar de sus límites en el uso asertivo o eficaz, les permite dar respuesta a las necesidades de la Universidad. Las deficiencias de los servicios de acceso a internet pueden que retrasen su migración a una Infocultura plena, que permita el desarrollo de redes, sin embargo, los usos de los dispositivos digitales posibilitan otros escenarios para la educación. Es necesario ajustar el uso de las tecnologías digitales a nuestros escenarios y procesos académicos, crear espacios físicos y digitales en conjunto permiten una pluralidad de acciones o experimentos inéditos; los cuales pueden limitarse si son centrados en la lógica de distribución de información. 
Vincular de manera asertiva la presencia de la Universidad en las redes sociales, les permitirá obtener un mayor alcance en la comunicación con todos los actores de la Extensión Académica, debido al contexto social presente en Venezuela. Además, permitirá percibir que las redes sociales son un sistema vivo y dispuesto a mantener a la comunidad universitaria informada de todas las acciones generadas, conjuntamente, sensibilizará sobre los problemas en pleno desarrollo. Del mismo modo, consolidar estos espacios son un punto estratégico, ya que son un elemento de influencia política y de movilización social que nos permite ser ubicuos en medio de urgencias.

En cuanto a las políticas implantadas por las autoridades gubernamentales respecto al uso del Software Libre, se percibe que no han sido tomadas debido a que las competencias ligadas a estos sistemas, no son representativos. No obstante, el empleo espontáneo para asumir paulatinamente políticas del Estado, limita las posibilidades a solo herramienta de apoyo en la labor diaria. Puede que investigaciones al respecto de la política, que manifiesten los beneficios que se generan sobre la apropiación de la cultura, sean necesarios y fundamentales. Igualmente, la gestión de políticas públicas en torno a las tecnologías digitales debe ir en función de los actores y su entorno, y el cómo se conjugan estos para adaptarse a los cambios.

Finalmente, estudios que tomen en cuenta la relación social con las TIL desde una perspectiva mixta, nos permitieron evidenciar la correlación del fenómeno desde diferentes perspectivas metodológicas. Este escenario nos permitió ampliar el análisis de los datos producidos, ya que colaboro en la profundización y comprensión de los fenómenos educativos, más allá de lo obvio. Así constituimos posibilidades para la Extensión Académica, que a partir de la comprensión a sus dinámicas podrán generar acciones al respecto. De igual modo, estas discusiones manifestaron de algún modo la relación con otros contextos en América Latina, lo que otorga la posibilidad a nuevas investigaciones el poder profundizar y comprender de mejor manera fenómeno semejantes a la situación estudiada.

\section{REFERENCIAS}

BONILLA, Maria Helena. A presença da cultura digital no GT Educação e Comunicação da ANPEd. Revista Teias, [S.1.], v. 13, n. 30, p. 23, dez. 2012. Recuperado de: https://www.epublicacoes.uerj.br/index.php/revistateias/article/view/24272/17251. Acceso el: 20 oct. 2018.

BONILLA, Maria Helena; PRETTO, Nelson De Luca. Apresentação. Em Aberto: Movimentos colaborativos, tecnologias digitais e educação, v. 28, n. N 94, p. 15-20, dez. 2015.

CASTELLANOS-AGUIRRE, Uriel; PRETTO, Nelson De Luca. Desafíos de las políticas educativas con acceso abierto en las Universidades Nacionales de Venezuela. In: Educaşão pública e pesquisa: Ataques, lutas e resistências. Ninterói - RJ: ANPED, 2019. p. 7. Recuperado de: http://39.reuniao.anped.org.br/wp-content/uploads/sites/3/trabalhos/4536-

TEXTO PROPOSTA COMPLETO.pdf. Acceso el: 2 dez. 2019.

CASTELLS, Manuel. A era da informação: economia, sociedade e cultura: A sociedade em rede. 18. ed. São Paulo: Tera e Paz, 2017.

MUÑOZ-CANO, Juan; CÓRDOVA, Juan; PRIEGO, Heberto. Dificultades y facilidades para el desarrollo de un proceso de innovación educativa con base en las Tecnologías de la Información y Comunicación (TIC). Formación universitaria, v. 5, n. 1, p. 3-12, 2012. Recuperado de: https://bit.ly/2TCVFqp. Acceso el: 20 oct. 2018. 
DOI: $10.12957 /$ teias. $\%$ Y.55438

MONTOYA-GONZÁLEZ, Yaimar Del Valle; BONILLA, Maria Helena. Aproximaciones a la cultura digital de las familias venezolanas en torno al proyecto Canaima Educativo. Contratexto, n. 32, p. 259-278, 22 nov. 2019.

HERNÁNDEZ, Roberto; FERNÁNDEZ, Carlos; BAPTISTA, María. Metodología de la investigación. 5. ed. México: D.F.: Mcgraw-hill, 2010.

VENEZUELA. Ley de Infogobierno. [S.l: s.n.]. Recuperado de: http://www.asambleanacional.gob.ve/documentos leyes/ley-de-infogobierno.pdf. Acceso el: 20 out. 2018, 17 sept. 2013.

MENEZES, Karina; PRETTO, Nelson De Luca. Pirâmide da Pedagogia Hacker: de sonhos coletivos a engajamentos reais. Revista Teias, v. 20, n. Esp, p. 148-166, 25 nov. 2019.

VENEZUELA. Estadisticas de migración 2012. [S.l: s.n.]. Recuperado de: https://softwarelibre.gob.ve/images/stories/pdf/estadisticamigracion 2012.pdf. Acceso el: 20 oct. 2017, 2012.

PRETTO, Nelson De Luca. Uma escola sem/com futuro: educação e multimidia. 8. ed. Salvador: EDUFBA, 2013.

PRETTO, Nelson De Luca. Educações, culturas e hackers: escritos e reflexões. 1. ed. Salvador: EDUFBA, 2017.

PROGRAMA, de las Naciones Unidas para el Desarrollo. Las tecnologías de la información y la comunicación al servicio del desarrollo. Caracas: Intenso Offset, 2002, 249 p. Recuperado de: https://bit.ly/2IWrPXB. Acceso el: 20 oct. 2017.

RAMÍREZ, María. Recursos tecnológicos para el aprendizaje móvil (Mlearning) y su relación con los ambientes de educación a distancia: implementaciones e investigaciones. Revista Iberoamericana de Educación a Distancia, v. 12, n. 2, p. 57-82, dez. 2009.

RUIZ, Bolívar. Instrumentos de investigación educativa. Barquisimeto: Tipografía y Litografía Horizontes, 2002. $2^{\mathrm{a}} \mathrm{Ed}$.

SANDOVAL, Carlos. Especialización en Teoria, Métodos y Técnicas de Investigación Social. Bogotá, Colombia: ARFO Editores e Impresores Ltda, 2002.

Submetido em outubro de 2020 Aprovado em marco de 2021

\section{Informações do(a)(s) autor(a)(es)}

Uriel José Castellanos Aguirre

Universidade Federal da Bahia

E-mail: urielcastellanos@gmail.com

ORCID: http://orcid.org/0000-0002-7811-5874

Link Lattes: http://lattes.cnpq.br/6479213510946258http://lattes.cnpq.br/6479213510946258 